\title{
A rapid method for infectivity titration of Andes hantavirus using flow cytometry
}

Gonzalo P. Barriga ${ }^{a}$, Constanza Martínez-Valdebenito ${ }^{b}$, Héctor Galeno ${ }^{c}$, Marcela Ferrés $^{\mathrm{b}}$, Pierre-Yves Lozach ${ }^{\mathrm{d}}$ and Nicole D. Tischler ${ }^{\mathrm{a}, \mathrm{e}^{*}}$

${ }^{a}$ Fundación Ciencia \& Vida, Molecular Virology Laboratory, Av. Zanartu 1482, Santiago, Chile.

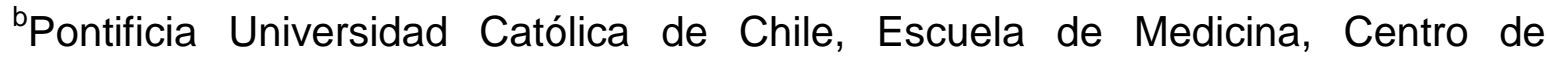
Investigaciones Médicas, División de Pediatría, Laboratorio de Infectología y Virología, Marcoleta 391, Santiago, Chile.

'Instituto de Salud Pública de Chile, Av. Marathon 1000, Santiago, Chile.

'INRS-Institut Armand-Frappier, Université du Québec, 531 boulevard des Prairies, Québec, Canada.

eUniversidad Andrés Bello, Facultad de Ciencias Biológicas, República 252, Santiago, Chile.

\section{Words Summary: 198}

Words Text: 1219

\section{"Corresponding author:}

Postal address: Av. Zanartu 1482, 7780272 Nunoa, Santiago, Chile. Phone: (56 2) 23672015. Fax: (56 2) 2372259.

E-mail address: ntischler@cienciavida.cl; tischlernd@gmail.com 


\section{Summary}

The focus assay is currently the most used technique for hantavirus titer determination. This method requires an incubation time between 5 to 11 days to allow the appearance of foci after several rounds of viral infection. The following work presents a rapid Andes virus (ANDV) titration assay, based on viral nucleocapsid protein $(\mathrm{N})$ detection in infected cells by flow cytometry. To this end, an anti-N monoclonal antibody was used that was developed and characterized previously. ANDV $\mathrm{N}$ could be detected as early as 6 hours post-infection, while viral release was not observed until 24 to 48 hours post-infection. Given that ANDV detection was performed during its first round of infection, a time reduction for titer determination was possible and provided results in only two days. The viral titer was calculated from the percentage of $\mathrm{N}$ positive cells and agreed with focus assay titers. Further, the assay was applied to quantify inhibition of ANDV cell entry by patient sera and by preventing endosome acidification. This novel hantavirus titration assay is a highly quantitative and sensitive tool that facilitates infectivity titration of virus stocks, rapid screening for antiviral drugs, and may be further used to detect and quantify infectious virus in human samples.

Keywords: Hantavirus, nucleoprotein, infectious virus titration, focus assay, flow cytometry 


\section{Short communication}

Hantaviruses are enveloped viruses with worldwide distribution that may cause severe diseases in humans (Krautkrämer et al., 2013; Macneil et al., 2011; Schmaljohn and Hjelle, 1997). Hantaviruses have a trisegmented, negative-sense single-strand RNA genome that encodes four structural proteins: the nucleocapsid protein $(\mathrm{N})$, the RNA dependent RNA polymerase and the $\mathrm{Gn}$ and $\mathrm{Gc}$ glycoproteins (Elliott, 1990). The viral $N$ protein is produced abundantly in hantavirus infected cells and can be detected as early as 4 hours (hrs) post-infection (Ramanathan et al., 2007; Schmaljohn and Hooper, 2001). Altogether, these features account for the main reason of hantavirus $\mathrm{N}$ protein detection for endpoint ELISA or immunofluorescence microscopy assays to assess the amount of viral particles and the progression of viral infection (Alexeyev et al., 1996). The detection of $N$ is also used to quantify the amount of infectious particles in focus assays (Bharadwaj et al., 2000; McCormick et al., 1982; Niklasson et al., 1991; Tanishita et al., 1984; Tischler et al., 2005). This last assay employs a semisolid overlay that is applied after virus adsorption, in order to limit virus replication and spread to the initial sites of infection, forcing the virus growth in foci. As hantaviruses are non-lytic in cell cultures in vitro, the foci are visualized by immunostaining with specific monoclonal or polyclonal antibodies against $\mathrm{N}$ in combination with immunocytochemical or immunofluorescence methods. Each focus represents an infective unit (IU) of the virus that has been produced by several cycles of infection and re-infection. In the case of hantaviruses, foci can be detected unambiguously between 5 to 11 days after infection, depending on the virus isolate (Heider et al., 2001; Tanishita et al., 1984). While the focus assay has been improved by use of immunodetection through chemiluminescence substrates (Heider et al., 2001), the quantitation of hantavirus infectivity by such an approach remains tedious, not accurate, and restricted to small scale diagnostics.

The present investigation conveys a simple, rapid and efficient assay to titer accurately the infectivity of Andes hantavirus (ANDV). This virus is located mainly 
in Argentina and Chile and has the particularity that it may spread through interhuman transmission. Further, it has been depicted as the only hantavirus for which an animal model with human-like disease is available (Ferres et al., 2007; Hooper et al., 2001; Martinez et al., 2005; Padula et al., 1998). The present work aims towards ANDV $\mathrm{N}$ protein immunodetection in infected cells by flow cytometry. Monoclonal antibodies (mAbs) against $N$ were prepared previously and their epitopes and cross-reactivity with other hantaviruses characterized (Tischler et al., 2008). The mAb 7B3/F7 was selected as it has been used successfully in focus assays, focus reduction neutralization assays and immunofluorescence assays of infected cells (Godoy et al., 2009; Vera-Otarola et al., 2012 and unpublished data). All procedures with infectious ANDV were performed under biosafety level 3 conditions. For ANDV titration, the human isolate ANDV strain Chi-7913 was used (Galeno et al., 2002). The protocol was performed as follows: ANDV were adsorbed for $1 \mathrm{hr}$ at $37^{\circ} \mathrm{C}$ to Vero E6 cells (ATTC CRL-1586) grown to $90 \%$ confluence in 6 well plates. Further, the cells were washed twice with PBS to remove non-adsorbed virus and incubated subsequently with DMEM supplemented with $10 \%$ fetal bovine serum (FBS). The viral infection was allowed to proceed for $6 \mathrm{hrs}$. At this time point, a feasible detection of $\mathrm{N}$ protein synthesis in infected cells was expected before the release of viral progeny. After this incubation period, cells were harvested by trypsinization. It is very likely that the cell trypsinization further accounts for removal of any virus that has not been uptaken by the cells. The infected cell suspension was collected in $15 \mathrm{ml}$ tubes containing $4 \%$ paraformaldehyde and incubated during $30 \mathrm{~min}$ at room temperature (RT). This fixation process inactivates ANDV in cell culture. Subsequently, the cells were washed with PBS and permeabilized by resuspension in PBS containing Triton X-100 $0.05 \%$. After $30 \mathrm{~min}$, the cells were washed with PBS and incubated for 45 min at RT with the mAb anti-N 7B3/F7 in a 1:5,000 dilution. After washing twice with PBS, primary antibody detection was performed with AlexaFluor 555 conjugated anti-mouse immunoglobulin (Invitrogen). After $30 \mathrm{~min}$ of incubation in the dark, the cells were washed and 
finally re-suspended in $400 \mu \mathrm{L}$ PBS and placed on FACS tubes for subsequent flow cytometry analysis (BD FACSCanto II). Mock-infected cells were treated equally and used for gating. 10,000 cells were counted from each condition. In Fig. 1A, typical titration results can be observed in which serial dilutions of ANDV inoculum were used to infect cells. N-positive cells were distinguished clearly from mockinfected control cells.

The viral production was assessed between 5 and $72 \mathrm{hrs}$ following the exposition of cells to the virus. As seen in Fig. 1B, viral progeny release occurred only after 24 hrs. This data confirms that no viral progeny was produced at $6 \mathrm{hrs}$ post-infection and that the flow cytometry-based titration assay measured the first round of ANDV infection. This detection sensitivity clearly benefits this assay in terms of its velocity. Although both cytometry and focus assays, cytomentry and focus assays start with identical virus infection times of 1 hour, the flow cytometry--based assay allows stopping virus incubation within the same day after $6 \mathrm{hrs}$. In contrast, the focus assay requires 5-11 days of virus incubation depending on the hantavirus. After discontinuing virus incubation by fixation of cells, an additional day is required for both assays for antibody staining and focus or flow cytometry analysis, respectively.

The detection of $\mathrm{N}$ protein has been used traditionally for hantavirus diagnostics and titration despite the fact that it is a structural component of the virus. In order to test if the flow cytometry titration assay enables the detection of a productive infection, and not just bound particles, Vero E6 cells were incubated for $1 \mathrm{hr}$ with different dilutions of untreated ANDV or heat-inactivated ANDV. Heat-inactivation of ANDV was performed at $56 \stackrel{\circ}{\circ}$ during $30 \mathrm{~min}$ as previously described for hantaviruses_(ref). Six hrs post-adsorption, infected Vero E6 cells were subjected to the detection of the $\mathrm{N}$ protein by flow cytometry analysis. Fig. $1 \mathrm{C}$ shows that infection of cells increased proportionally to the amount of untreated virus. In contrast, no increase was observed when cells were incubated with equivalent 
amounts of heat-inactivated ANDV. Instead, a signal below $1 \%$ of total cells was detected, reflecting the assay background (Fig 1C). This data confirmed that the signal associated with $\mathrm{N}$ detection accounts for viral infection and replication rather than viral input.

To test if the developed flow cytometry assay allowed reliable titration of virus stocks, different batches and dilutions thereof were analyzed and compared with results obtained by traditional focus assay (Tischler et al., 2005). Virus titers obtained from flow cytometry titration assays were calculated within the linear range spanning values between 1 and $40 \%$ of infected cells, similar to the range established for other viruses (Drayman and Oppenheim, 2011). The following formula was used for the calculation of titers expressed in infectious units $/ \mathrm{ml}$ :

$$
\text { Titer }=\frac{N \times I C[\%]}{V[m L] \times 100}
$$

$\mathrm{N}$ corresponds to the number of cells used for initial infection, IC to the percentage of infected cells and $V$ to the volume $(\mathrm{ml})$ of virus stock used for infection. As can be seen in Fig. 2A, the results of both assays were in good agreement over the whole range of tested virus titers, revealing values which were within the standard deviation of each assay (Fig. 2A).

Finally, the flow cytometry titration assay was challenged in hantavirus inhibition studies. For this purpose, ANDV was pre-incubated with different sera derived from ANDV infected patients. These sera were characterized previously concerning their activity to neutralize ANDV and simian immunodeficiency virus particles pseudotyped with ANDV glycoproteins (Tischler et al., 2005; Cifuentes-Muñoz et al., 2010). Each of the tested patient serum diminished virus infection over $75 \%$ while viral infectivity was not reduced by the negative control serum (Fig. 2B). ANDV infection of cells was further blocked by different concentrations of ammonium chloride, a drug weak base that prevents acidification of endosomes. A low $\mathrm{pH}$ endosome $\mathrm{pH}$ is required for virus:cell the fusion of virus and endosome 
membranes fusion-during cell entry of hantaviruses and other bunyaviruses after viral uptake through receptor mediated endocytosis (Hacker and Hardy, 1997; Jin et al., 2002; Lozach et al., 2010; Pekosz et al., 1995). Ammonium chloride was added in different concentrations to cells prior to infection and during incubation with ANDV. As seen in Fig. 2C, increasing amounts of ammonium chloride reached up to $97 \%$ of inhibition in a dose-dependent manner. Altogether, these ANDV inhibition experiments demonstrate that this assay is highly suited for rapid testing of hantavirus inhibitors.

Alternative systems to screen for virus neutralization have been developed based on virus particles pseudotyped with ANDV surface glycoproteins (Cifuentes-Muñoz et al., 2010; Ray et al., 2010). Although these systems provide the advantage to circumvent the use of BSL-3 safety requirements, their use is reduced to screening of compounds that act on virus cell entry and do not allow the selection of inhibitors that direct later virus replication steps.

Taken together, in this work a novel hantavirus titration assay was developed using flow cytometry. The current assay was performed in 6 well plates and can be upscaled easily to a higher well format. The new method presents several advantages compared to those established previously. It is fast, quantitative and can be adapted for high-throughput measurements. Although this assay was used for ANDV titration, it may be applied to other hantaviruses. In conclusion, this novel assay allows rapid titration of virus stocks, screening for antiviral drugs and may be further useful in direct detection of infectious virus in human samples.

\section{Acknowledgments}

This work was financed by CONICYT through grant FONDECYT 1100756 and CONICYT basal funding PFB-16. 


\section{Literature}

Alexeyev, O.A., Elgh, F., Ahlm, C., Stigbrand, T., Settergren, B., Wadell, G., Juto, P., 1996. Hantavirus antigen detection using human serum immunoglobulin $M$ as the capturing antibody in an enzyme-linked immunosorbent assay. Am. J. Trop. Med. Hyg. 54, 367-71.

Bharadwaj, M., Nofchissey, R., Goade, D., Koster, F., Hjelle, B., 2000. Humoral immune responses in the hantavirus cardiopulmonary syndrome. J. Infect. Dis. 182, 43-8.

Cifuentes-Muñoz, N., Darlix, J.L., Tischler, N.D., 2010. Development of a lentiviral vector system to study the role of the Andes virus glycoproteins. Virus Res. 153, 29-35.

Drayman, N., Oppenheim, A., 2011. Rapid titration of viruses by flow cytometry. Curr. Protoc. Cell Biol. 51, 26.11.1-26.11.7.

Elliott, R.M., 1990. Molecular biology of the Bunyaviridae. J. Gen. Virol. 71, 501-22.

Ferres, M., Vial, P., Marco, C., Yanez, L., Godoy, P., Castillo, C., Hjelle, B., Delgado, I., Lee, S.J., Mertz, G.J., 2007. Prospective evaluation of household contacts of persons with hantavirus cardiopulmonary syndrome in Chile. J. Infect. Dis. 195, 1563-71.

Galeno, H., Mora, J., Villagra, E., Fernandez, J., Hernandez, J., Mertz, G.J., Ramirez, E., 2002. First human isolate of Hantavirus (Andes virus) in the Americas. Emerg. Infect. Dis. 8, 657-61.

Godoy, P., Marsac, D., Stefas, E., Ferrer, P., Tischler, N.D., Pino, K., Ramdohr, P., Vial, P., Valenzuela, P.D., Ferres, M., Veas, F., Lopez-Lastra, M., 2009. Andes virus antigens are shed in urine of patients with acute hantavirus cardiopulmonary syndrome. J. Virol. 83, 5046-55.

Hacker, J.K., Hardy, J.L., 1997. Adsorptive endocytosis of California encephalitis virus into mosquito and mammalian cells: a role for G1. Virology 235, 40-7.

Heider, H., Ziaja, B., Priemer, C., Lundkvist, A., Neyts, J., Kruger, D.H., Ulrich, R., 2001. A chemiluminescence detection method of hantaviral antigens in neutralisation assays and inhibitor studies. J. Virol. Methods 96, 17-23.

Hooper, J.W., Larsen, T., Custer, D.M., Schmaljohn, C.S., 2001. A lethal disease model for hantavirus pulmonary syndrome. Virology 289, 6-14.

Jin, M., Park, J., Lee, S., Park, B., Shin, J., Song, K.J., Ahn, T.I., Hwang, S.Y., Ahn, B.Y., Ahn, K., 2002. Hantaan virus enters cells by clathrin-dependent receptor-mediated endocytosis. Virology 294, 60-9.

Krautkrämer, E., Zeier, M., Plyusnin, A., 2013. Hantavirus infection: an emerging infectious disease causing acute renal failure. Kidney Int. 83, 23-7.

Lozach, P.Y., Mancini, R., Bitto, D., Meier, R., Oestereich, L., Overby, A.K., Pettersson, R.F., Helenius, A., 2010. Entry of bunyaviruses into mammalian cells. Cell Host Microbe 7, 488-99.

Macneil, A., Nichol, S.T., Spiropoulou, C.F., 2011. Hantavirus pulmonary syndrome. Virus Res. 162, 138-47. 
Martinez, V.P., Bellomo, C., San Juan, J., Pinna, D., Forlenza, R., Elder, M., Padula, P.J., 2005. Person-to-person transmission of Andes virus. Emerg. Infect. Dis. 11, 184853.

McCormick, J.B., Sasso, D.R., Palmer, E.L., Kiley, M.P., 1982. Morphological identification of the agent of Korean haemorrhagic fever (Hantaan virus) as a member of the Bunyaviridae. Lancet 1, 765-8.

Niklasson, B., Jonsson, M., Lundkvist, A., Horling, J., Tkachenko, E., 1991. Comparison of European isolates of viruses causing hemorrhagic fever with renal syndrome by a neutralization test. Am. J. Trop. Med. Hyg. 45, 660-5.

Padula, P.J., Edelstein, A., Miguel, S.D., Lopez, N.M., Rossi, C.M., Rabinovich, R.D., 1998. Hantavirus pulmonary syndrome outbreak in Argentina: molecular evidence for person-to-person transmission of Andes virus. Virology 241, 323-30.

Pekosz, A., Griot, C., Nathanson, N., Gonzalez-Scarano, F., 1995. Tropism of bunyaviruses: evidence for a G1 glycoprotein-mediated entry pathway common to the California serogroup. Virology 214, 339-48.

Ramanathan, H.N., Chung, D.H., Plane, S.J., Sztul, E., Chu, Y.K., Guttieri, M.C., McDowell, M., Ali, G., Jonsson, C.B., 2007. Dynein-dependent transport of the hantaan virus nucleocapsid protein to the endoplasmic reticulum-Golgi intermediate compartment. J. Virol. 81, 8634-47.

Ray, N., Whidby, J., Stewart, S., Hooper, J.W., Bertolotti-Ciarlet, A., 2010. Study of Andes virus entry and neutralization using a pseudovirion system. J. Virol. Methods 163, 416-23.

Schmaljohn, C., Hooper, J., 2001. Bunyaviridae: the viruses and their replication, in: Knipe, D.M., Howley, P.M., Griffin, D.E., Lamb, R.A., Martin, M.A., Roizman, B., Straus, S.E. (Eds.), Fields Virology, $4^{\text {th }}$ ed., vol 2. Lippincott Williams \& Wilkins, Philadelphia, PA.

Schmaljohn, C., Hjelle, B., 1997. Hantaviruses: a global disease problem. Emerg. Infect. Dis. 3:95-104.

Tanishita, O., Takahashi, Y., Okuno, Y., Yamanishi, K., Takahashi, M., 1984. Evaluation of focus reduction neutralization test with peroxidase-antiperoxidase staining technique for hemorrhagic fever with renal syndrome virus. J. Clin. Microbiol. 20, 1213-5.

Tischler, N.D., Galeno, H., Rosemblatt, M., Valenzuela, P.D., 2005. Human and rodent humoral immune responses to Andes virus structural proteins. Virology 334, 31926.

Tischler, N.D., Rosemblatt, M., Valenzuela, P.D., 2008. Characterization of cross-reactive and serotype-specific epitopes on the nucleocapsid proteins of hantaviruses. Virus Res. 135, 1-9.

Vera-Otarola, J., Solis, L., Soto-Rifo, R., Ricci, E.P., Pino, K., Tischler, N.D., Ohlmann, T., Darlix, J.L., Lopez-Lastra, M., 2012. The Andes hantavirus NSs protein is expressed from the viral small mRNA by a leaky scanning mechanism. J. Virol. 86, 2176-87. 


\section{Figure Legends}

Fig. 1: Flow cytometry detection of ANDV $\mathbf{N}$ positive cells. A) Flow cytometry plots resulting from Vero E6 cell infection in 6 well plates with serial dilutions of virus inoculums. The gate of $\mathrm{N}$ positive cells, termed gate $\mathrm{P} 4$, was established using mock-infected cells (upper left corner). The percentage of $\mathrm{N}$ positive cells is indicated in each graph within the established P4 gate. B) Viral progeny particle release kinetics. Vero E6 cells were infected with $\mathrm{MOI} 0.2$ and supernatants harvested at indicated time points. Infectious particles in these supernatants were tittered by subsequent infection of Vero $\mathrm{E} 6$ cells and detection of $\mathrm{N}$ positive cells after 6 hrs by flow cytometry. C) Titration of untreated and heat inactivated virus by flow cytometry. Vero E6 cells were incubated with different amounts of untreated virus or heat-inactivated virus. Six hrs post-infection, $\mathrm{N}$ positive cells were quantified by flow cytometry.

Fig. 2: ANDV stock titration and inhibition quantitation by flow cytometry assays. A) Comparison of virus titers from different stocks by flow cytometry using and focus assay using ANDV $\mathrm{N}$ for immunodetection. The focus assay was performed as previously described (Tischler et al., 2005). B) Neutralization of ANDV infectivity by sera of ANDV infected patients. ANDV was incubated for $1 \mathrm{~h}$ with sera from ANDV infected patients (1/50 dilution), human negative control serum or without serum. The virus-sera mixture was added to cells and incubated for 2 hours at $37^{\circ} \mathrm{C}$ ( $\left.\mathrm{MOI} 0.1\right)$. The viral infection was allowed to proceed for $6 \mathrm{hrs}$ after which it was quantified by flow cytometry. C) Inhibition of ANDV infectivity using different concentrations of ammonium chloride. Equal ANDV inoculums were added to Vero E6 cells treated with different concentrations of ammonium chloride. The percentage of infected cells was tittered by flow cytometry detecting ANDV N in infected cells. 
A
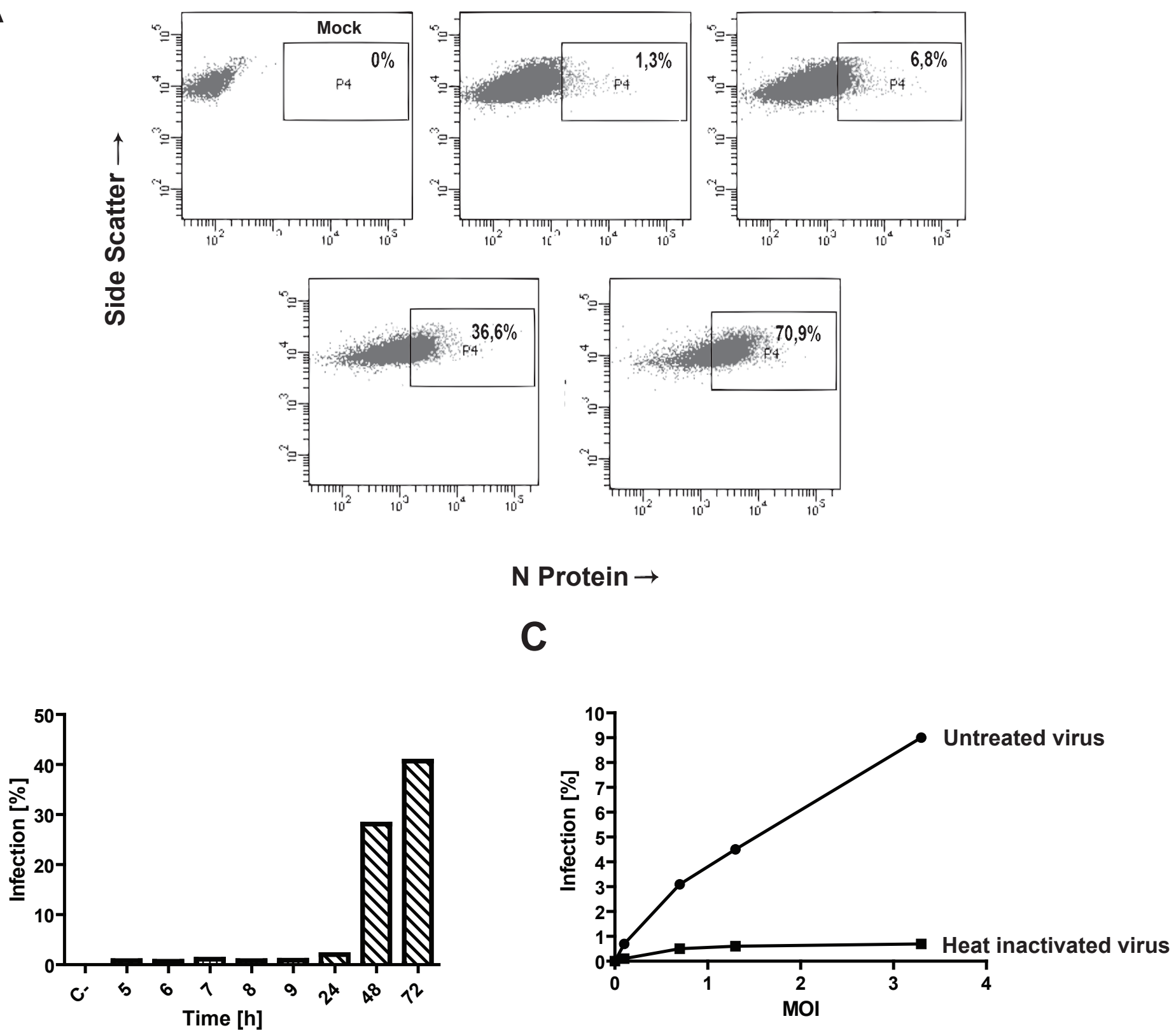
A
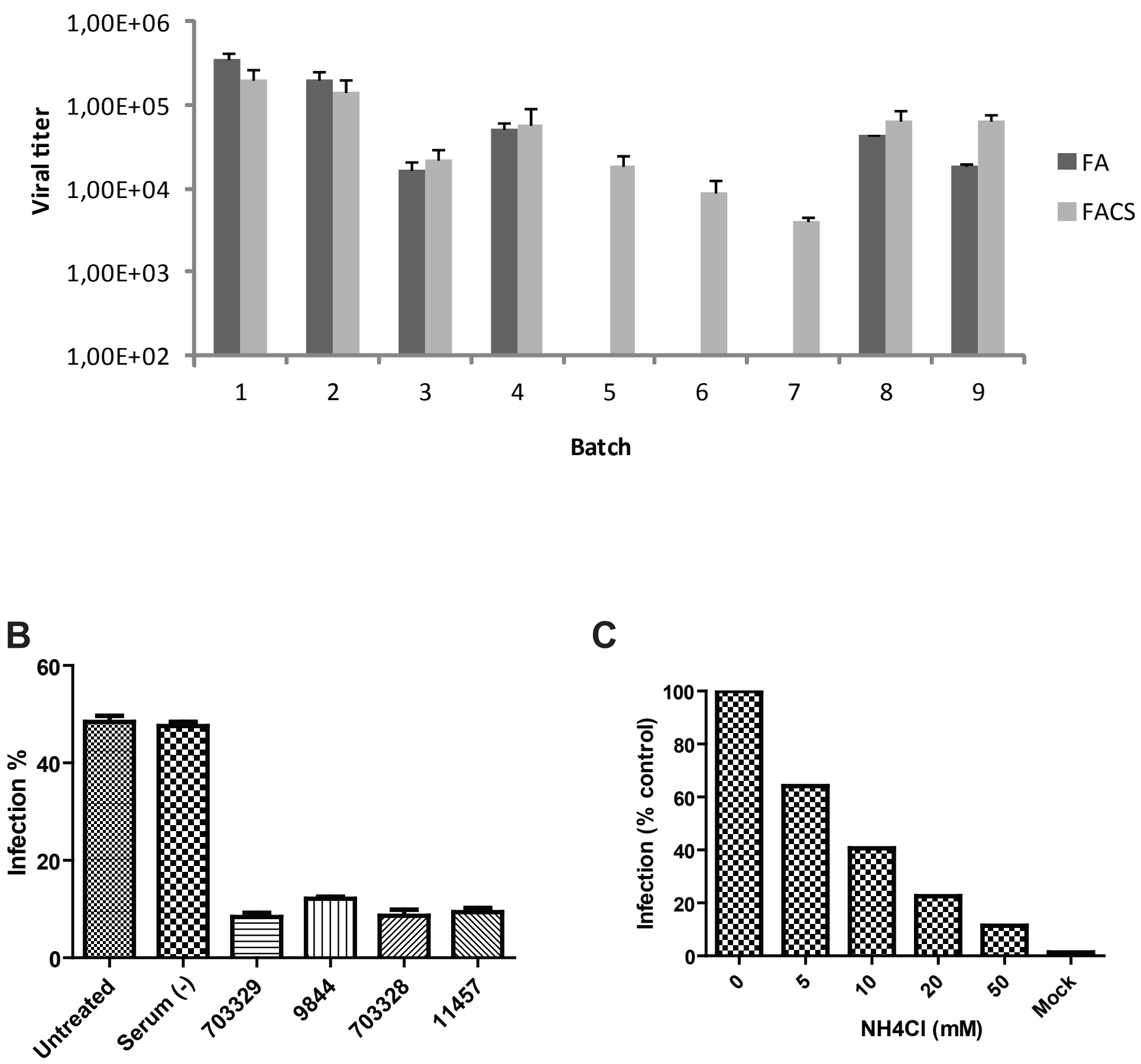

C

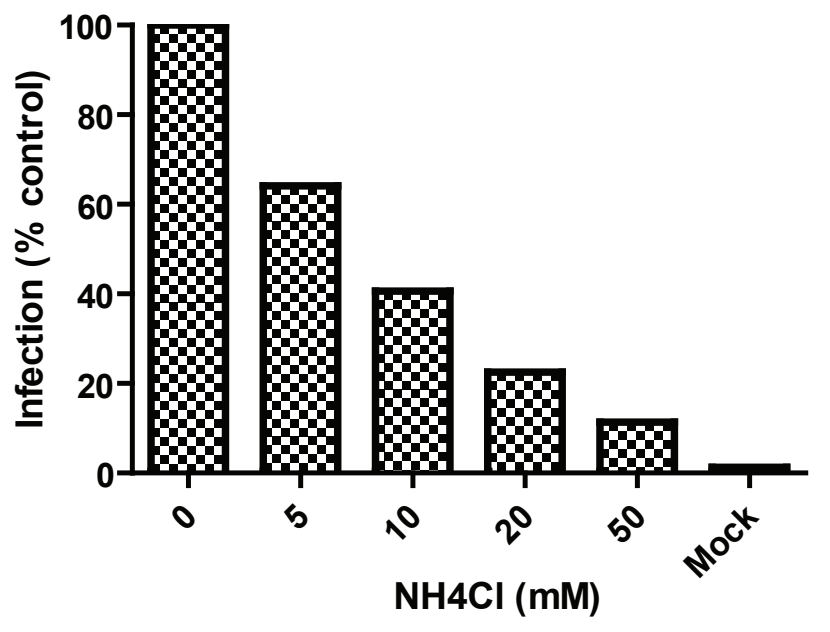

